\title{
A comparison between active and passive microwave measurements of the Antarctic ice sheet and their association with the surface katabatic winds
}

\author{
F. REMY AND J. F. MinsteR \\ UM 39, Groupe de Recherche de Géodésie Spatiale, 18 avenue Edouard-Belin, 31055 Toulouse Cedex, France
}

\begin{abstract}
The intensity of the Seasat altimeter return power over Antarctica varies in strong correlation with the intensity of model katabatic winds. It is also strongly correlated with the polarization of the passive microwave signal at $37 \mathrm{GHz}$ of the Nimbus-7 SMMR data. It is shown that this is most likely the result of the windinduced micro-roughness of the ice surface.
\end{abstract}

\section{INTRODUCTION}

The main interest in satellite-altimetry measurements above continental ice so far has been the construction of a very precise surface topography (Zwally and others, 1983; Remy and others, 1989). Recently, Ridley and Partington (1988) and Partington and others (1989) have suggested that the radar pulse penetrates into dry snow and that the return signal is affected by a so-called volume scattering. In this case, the height as measured by the radar is below the actual surface, and the possibility of surveying the Greenland and Antarctic mass budgets by repeated mapping of the topography becomes questionable: variations of altimetric height could result from variations of ice temperature, density or grain-size, which affect the volume scattering.

It is therefore necessary to quantify the respective importance of volume and surface scattering accounting for the altimeter return signal. A direct analysis of the altimeter return wave forms, as done by Ridley and Partington (1988) or Partington and others (1989) seems to be very difficult; a large number of unknown parameters have to be considered (satellite pointing angle, surface slope, medium- and large-scale surface features, ice temperature, density and grain-size). Also, precise modelling of microwave propagation in dry snow is limited by the uncertainty of the imaginary part of the dielectric constant (Matzler, 1987).

Recently, Remy and others (1990) analysed the intensity of the Seasat altimeter return signal; variations of up to $15 \mathrm{~dB}$ were observed, corresponding to variations by a factor of 30 on the back-scatter coefficient. In addition, this signal is highly correlated with katabatic wind intensity. It is due either to surface micro-roughness in the case of a pure surface scattering, or to snow grain- size, in the case of a pure volume scattering. The latter case is not easily supported by in-situ data because it would require variations of grain-size much larger than observed. Moreover, if a katabatic wind produces such grain-size variations, its effect would also be visible on the accumulation-rate maps, derived from passive microwave measurements as done by Rotman and others (1982), which is not the case. On the contrary, in the case of a pure surface scattering, micro-roughness variations as derived from altimeter return-power variations are consistent with observations.

The aim of this note is to analyse this question further by comparing the intensity of the Seasat altimetric return signal with the brightness temperature at $37 \mathrm{GHz}$ as measured by the passive microwave radiometer SMMR (Scanning Multichannel Microwave Radiometer) on board Nimbus-7. The latter signal is known to be dominantly affected by volume scattering (Zwally, 1977) and by layering (Matzler and others, 1984), though it is also sensitive to surface roughness (e.g. Tsang and Newton, 1982). Note that passive microwave radiometry is actually the best technique to measure, on a large scale, the accumulation rate, which is a poorly known yet essential parameter of continental ice-sheet evolution. Therefore, it is important to assess its sensitivity to surface roughness.

The approach is semi-empirical; we examine the correlation of the various signals and compare the order of magnitude of the possible surface and volume effects with the observed variations.

\section{ENERGY RETURN}

We used the Seasat altimeter-sensor data records. They 


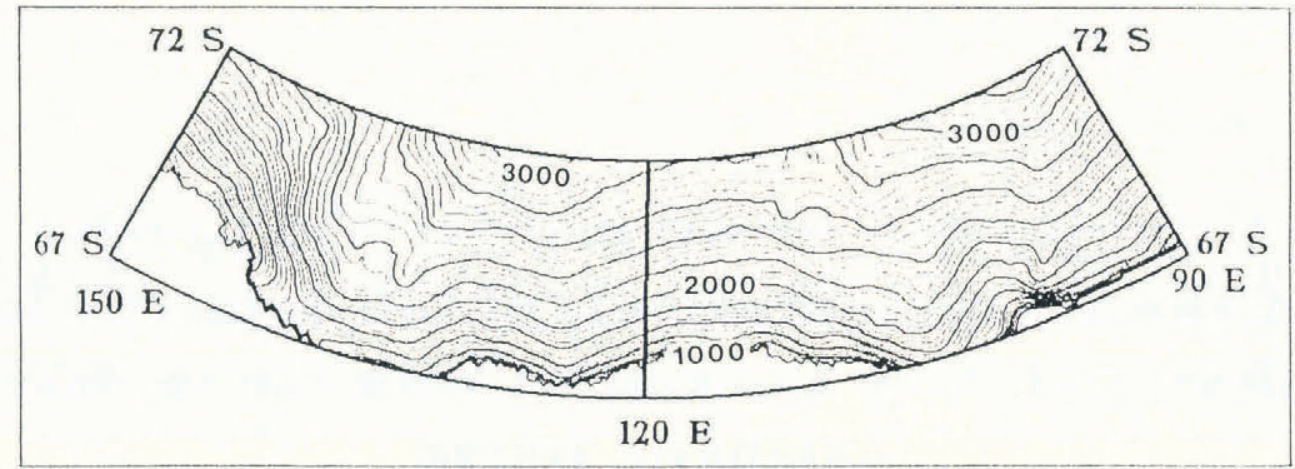

Fig. 1. Topographic map of the selected sector of Antarctica, deduced from Seasat data, as explained in Remy and others (1989). Bold isolines are each $200 \mathrm{~m}$, thin isolines are each $50 \mathrm{~m}$.

are provided every $0.1 \mathrm{~s}$, that is every $700 \mathrm{~m}$ along the satellite track. They are first corrected and normalized, so that the mean power intensity is positioned at the middle of the radar receiving window (Remy and others, 1990). Then the intensity of the return signal is given by the corrected Automatic Gain Control (AGC). In order to compare these altimetric data with the SMMR data, the AGC values are then averaged over domains defined by the SMMR cells $(25 \mathrm{~km} \times 25 \mathrm{~km}$ resolution for $37 \mathrm{GHz})$. This process smooths the medium-scale signal of the energy return (Remy and others, 1990). The selected region for the comparison (Terre Adélie and Wilkes Land) is limited by the Seasat data set (to the north of $72^{\circ} \mathrm{S}$, between $150^{\circ}$ and $90^{\circ} \mathrm{E}$ ), and corresponds to about 1250 cells. The altitude of the ice surface in this area is between 1000 and $3500 \mathrm{~m}$ (Fig. 1), half of our data being in the $2500-3000 \mathrm{~m}$ range. Thus, the ice surface will be assumed to be composed of dry snow. The model katabatic windflow lines of Parish (1982) are shown in Figure 2. This wind, being mostly related to the topography, is quite persistent both in strength and direction, and can be seen as a climatological wind.

As shown by comparison with Figure $3 \mathrm{a}$, which presents AGC values from the Seasat altimeter, the large-scale katabatic wind pattern is strongly correlated with large-scale variations of AGC, averaged for the cells. After digitalization of the flow lines, Remy and others (1990) found a correlation coefficient of 0.6 between the two fields.

The AGC, given in $\mathrm{dB}$, can be written as

$$
\mathrm{AGC}=\kappa_{\mathrm{dB}}+10 \log \left(\sigma_{0}\right)
$$

where $K_{\mathrm{dB}}$ is a system constant $(22 \mathrm{~dB})$ and $\sigma_{0}$ is the backscatter coefficient. If the echo is a surface scattering, then (Fung and Eom, 1982):

$$
\sigma_{\text {sur }}=R^{2} /\left(2 S^{2}\right)
$$

where $R$ is the Fresnel reflection coefficient at normal incidence, which is only dependent on the snow density for dry snow. $S^{2}$ is the variance of the surface slope, which can be expressed as a micro-roughness parameter. In this case, Figure $3 \mathrm{a}$ is easily explained; strong katabatic winds would induce increased micro-roughness, which would decrease the return energy.

On the other hand, AGC can be affected by volume scattering (Ridley and Partington, 1988). In this case, the back-scatter coefficient is mostly dependent on the scattering coefficient $k_{\mathrm{s}}$ (Remy and others, 1990) and can be written as:

$$
\sigma_{\mathrm{vol}}=a k_{\mathrm{s}}
$$

where $a$ is a first-order constant. $k_{\mathrm{s}}$ depends on the dielectric constant, temperature and density of snow, but its main variations are due to grain-size variations. Figure 3a should mostly result from effects of the wind on grainsize; according to Male (1980), the wind should break the snow grains, thus diminishing the volume scattering and in consequence the altimeter return energy. According to Remy and others (1990), this scheme cannot explain all the observed variations and cannot be the main factor affecting the altimetric return. However, one cannot assume it does not exist.

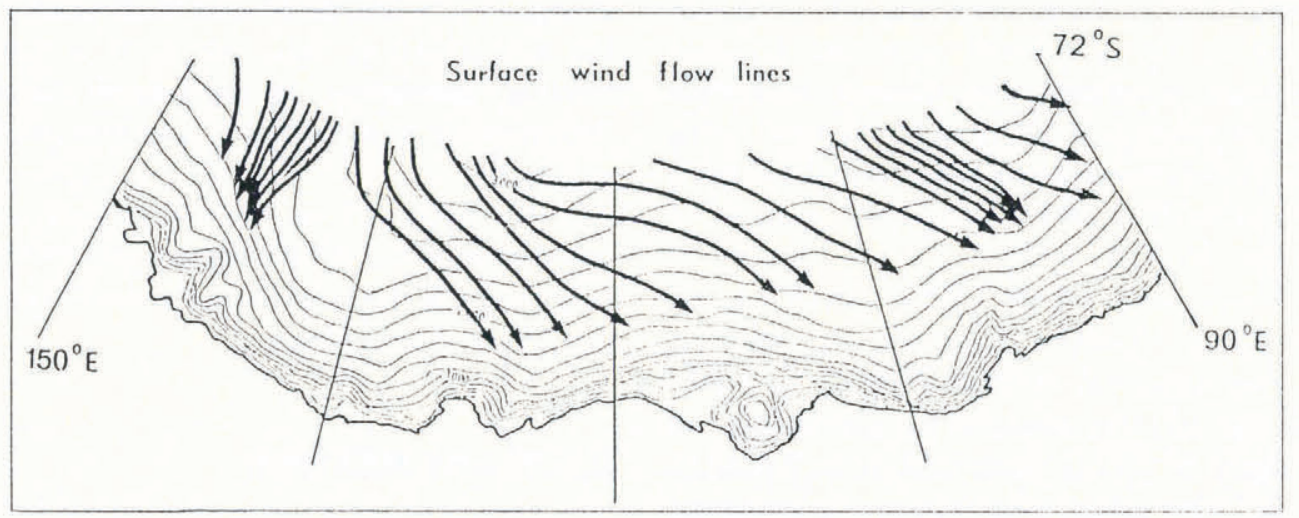

Fig. 2. Katabatic wind-flow lines of Parish (1982) superimposed on the topographic map of Drewry (1983). 


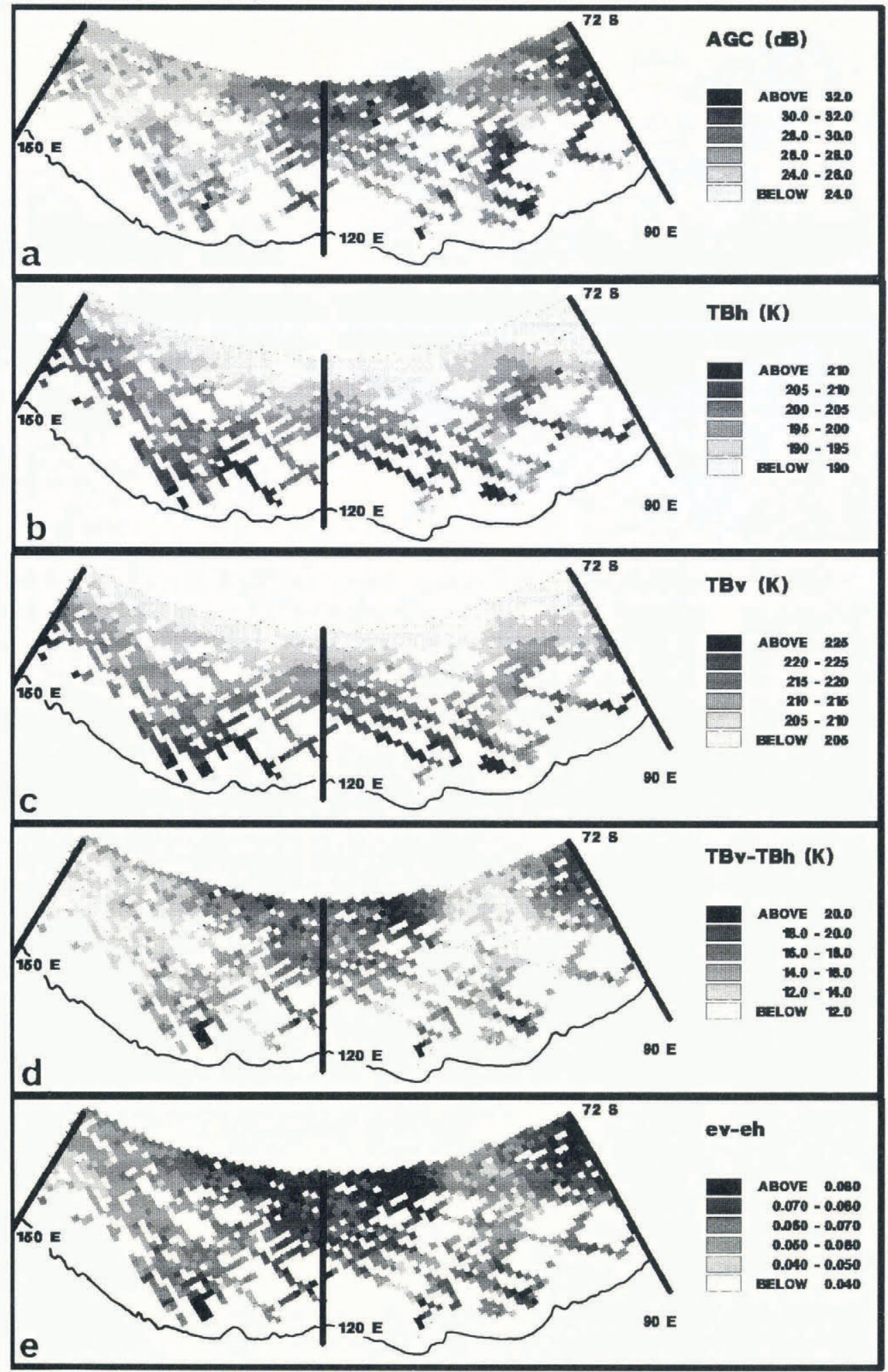

Fig. 3. Averaged values over $125025 \mathrm{~km} \times 25 \mathrm{~km}$ domains of the sector of Antarctica shown in Figure 1, for (a) AGC of the Seasat altimeter; (b) Brightness temperature at $37 \mathrm{GHz}$ for November 1979, deduced from Nimbus-7 SMMR data, for horizontal polarization; (c) Brightness temperature for vertical polarization; (d) Difference between brightness temperature for vertical and horizontal polarizations; (e) Difference between emissivity for vertical and horizontal polarizations.

\section{COMPARISON WITH BRIGHTNESS TEMPERATURE AND EMISSIVITY}

Figure $3 \mathrm{~b}$ and $\mathrm{c}$ show the brightness temperatures, at $37 \mathrm{GHz}$, deduced from the SMMR passive radiometer of Nimbus-7, for the horizontal (TBh) and the vertical (TBv) polarizations, respectively. These data were taken in late
November when the snow-surface temperature is close to the mean annual temperature (Lettau, 1969); this decreases the possible effects of seasonal fluctuations. Zwally (1977) showed that the volume scattering by snow crystals is the dominant factor affecting the microwave emission. To a first approximation, the snowcrystal size is mainly dependent on the in-situ tempera- 

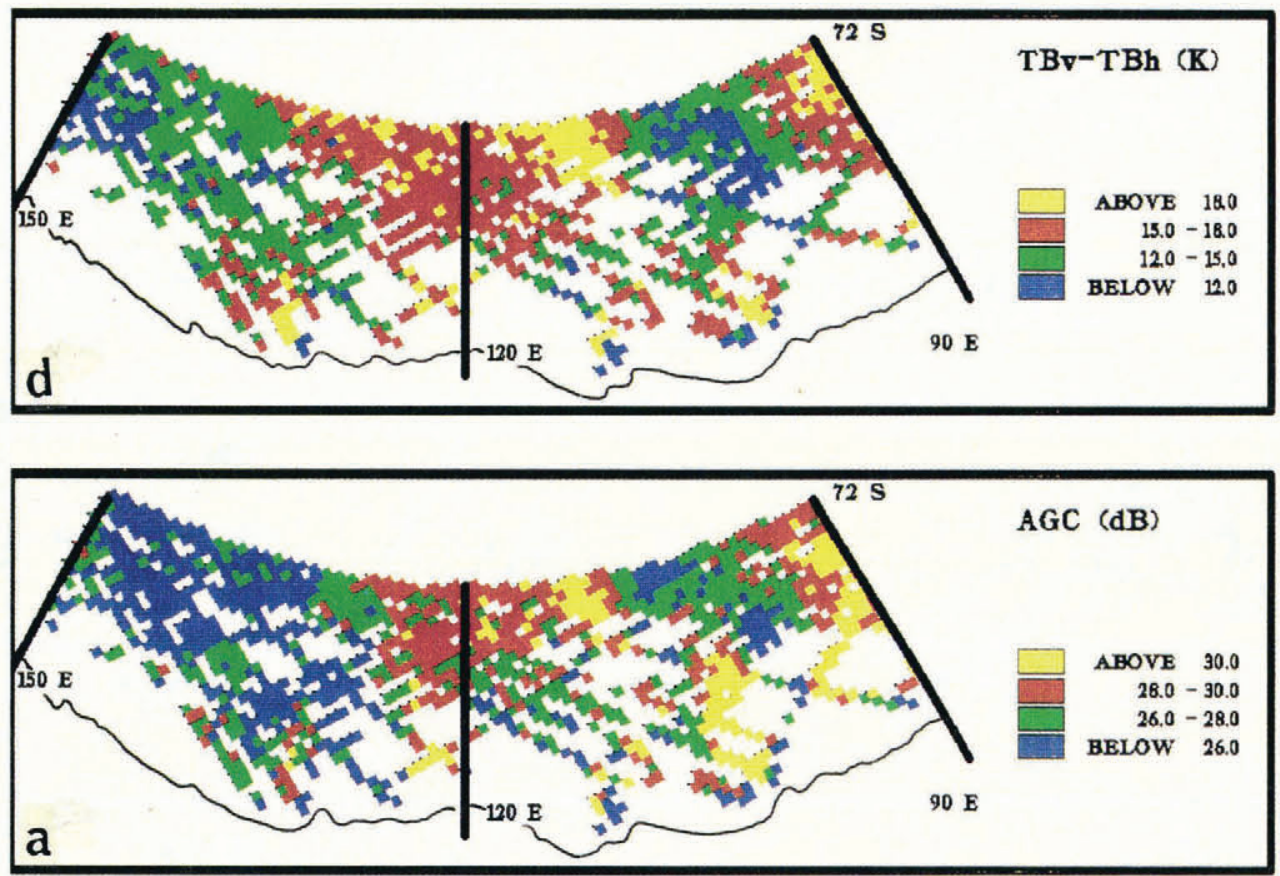

Fig. 3'. Colour representation of averaged values over $125025 \mathrm{~km} \times 25 \mathrm{~km}$ domains of the sector of Antarctica shown in Figure 1, for (a) AGC of the Seasat altimeter; (d) Differences between brightness temperature for vertical and horizontal polarizations.

ture, directly linked with altitude; this is evident if one compares Figure $3 \mathrm{~b}$ and $\mathrm{c}$ with Figure 1. The linear correlations between the brightness temperature for both polarization and altitude as derived from the altimeter (closely related to in-situ temperature) are 0.79 and 0.82 , respectively.

The first-order correlations between the brightness temperatures and AGC are poor: -0.11 and -0.28 for vertical and horizontal polarizations, respectively. Note that, at the $1 \%$ significance level, the value for a 1000 point sample is 0.08 .

On the other hand, the map of the difference between the two brightness temperatures (TBv-TBh; Fig. 3d) looks strongly like that for AGC. The regions where the wind is very strong (Fig. 3a; AGC $<28 \mathrm{~dB}$ ) show small polarization effects (less than $14 \mathrm{~K}$ ). Conversely, the regions where the wind is fair (AGC in Figure 3a is greater than $30 \mathrm{~dB}$ ) show important polarization effects $(>16 \mathrm{~K})$. Figure 3a and $\mathrm{d}$ are also shown in color.

Figure 4 shows binned temperature differences versus binned $\sigma_{0}$ values. Except for the strong $\sigma_{0}$ values, the relation between $\sigma_{0}$ and polarization is close to linear. The linear correlation coefficient, deduced from the 1250 points sample, between $\mathrm{TBv}-\mathrm{TBh}$ and $\sigma_{0}$ is 0.55 ; hence, about $30 \%$ of the variance of polarization is related to variations in $\sigma_{0}$. Note that, as for the AGC map, no visual correlation is observed between polarization and altitude. The correlation coefficient, $<0.02$, suggests that polarization cannot be created by a temperature-dependent parameter.

Finally, the correlations between $\mathrm{TBv}-\mathrm{TBh}$ and $\mathrm{TBv}$ or TBh are respectively 0.04 (less than the value at the $1 \%$ significance level) and 0.37 . Thus, most of the signal of $\mathrm{TBv}-\mathrm{TBh}$ comes from the horizontal polarization brightness temperature, which is known to be more variable than the vertical one (Matzler and others, 1984).
The emissivities, ev and eh, are given by the brightness temperature for both polarizations, normalized by the in-situ temperature $T p$. In late November, this temperature can be approximated by the annual mean air temperature as given by the empirical formula:

$$
\tau_{p}=258-H / 100(\mathrm{~K})
$$

where $H$ is the mean altitude of the cell in meters.

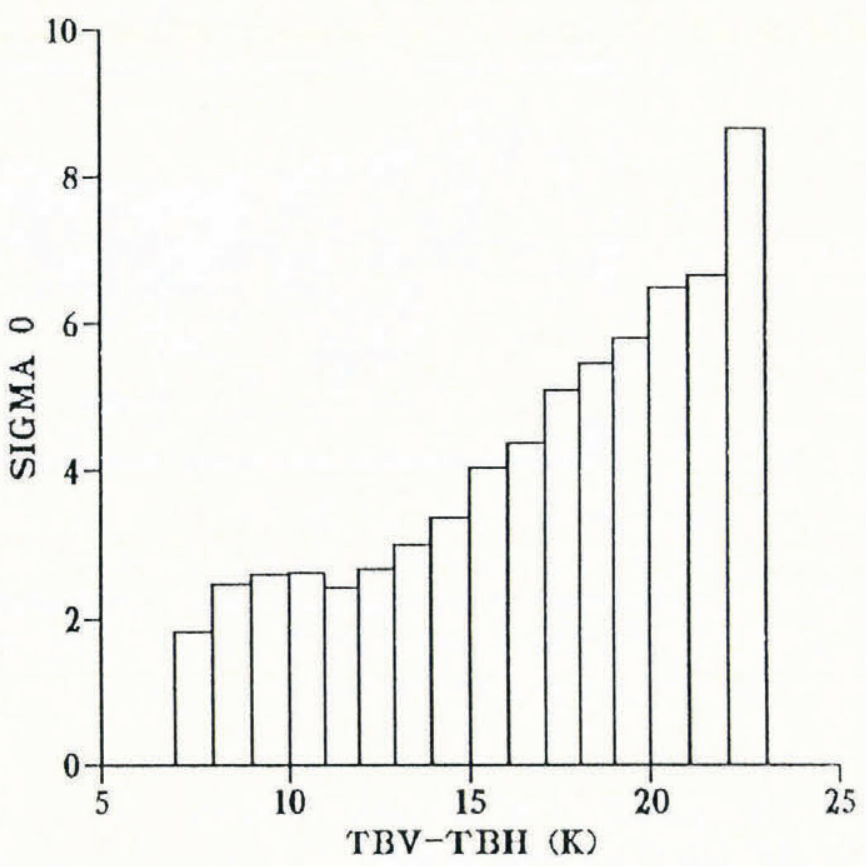

Fig. 4. Empirical relation between $\sigma_{0}$, the altimeter backscatter coefficient and the difference between vertical and horizontal polarizations of the brightness temperatures, averaged for each $1 \mathrm{~dB}$ interval of $\sigma_{0} . \sigma_{0}$ is related to AGC by Equation (1). 
For a mean temperature of $240 \mathrm{~K}$, a variation of $2 \mathrm{~K}$ leads to less than $1 \%$ variation in the deduced emissivity; the uncertainties in $T p$ have a negligible effect on emissivities.

Figure 3e shows the map for ev-eh, the difference between emissivities for vertical and horizontal polarizations; the variations are from 0.04 to 0.09 . The mean value is 0.065 and the variance is $(0.01)^{2}$. The correlations
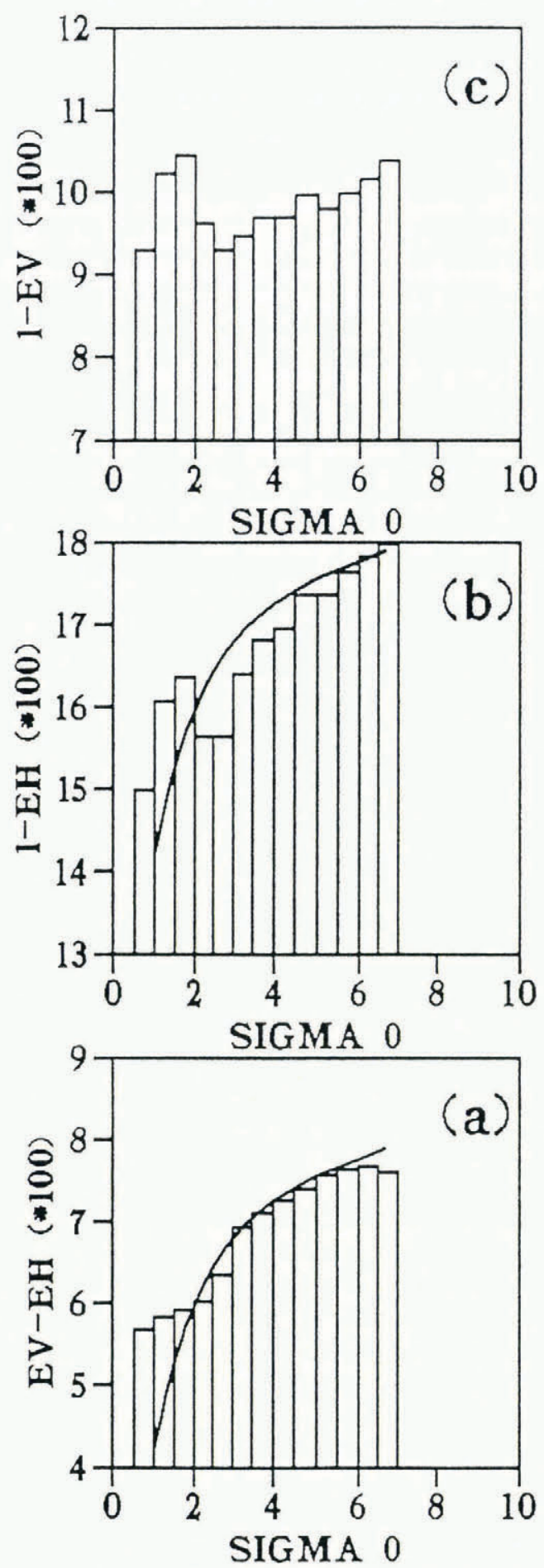

Fig. 5. Empirical relation between the altimetric back-scatter coefficient, averaged over each $0.5 \mathrm{~dB}$ interval and (a) ev-eh, (b) 1-eh, (c) 1-ev, where ev and eh are the emissivities in vertical and horizontal polarizations. The theoretical surfaceroughness effect on the emissivity (Equations (10)-(12) are superimposed. The roughness parameter $S^{2}$ is deduced from $\sigma_{0}$ of the Seasat altimeter (Equation (2)). between ev-eh and ev or eh, are respectively 0.01 and -0.5 ; the variations of ev-eh are mostly imputable to variations of eh.

The correlation coefficients between eh, ev, ev-eh and AGC are respectively $-0.32,0.06$, and 0.6 . Note that the correlation between AGC and the horizontal polarization signal is increased after removal of the altitude effect on temperature, while the correlation for vertical polarization is decreased.

Figure 5 shows the binned ev-eh, 1-eh and 1-ev values versus the altimetric back-scatter coefficient. Again, most of the emissivity variations and of the correlation with $\sigma_{0}$ are due to the horizontal polarization signal.

\section{DISCUSSION}

Variations in the polarization of emissivity have been described in relation to variations of the surface roughness (Choudhury and others, 1979; Tsang and Newton, 1982) and to variations of layering phenomena of the surface crust (Matzler and others, 1984; Stogryn, 1986). In both cases, these effects induce mostly variations in the signal in the horizontal polarization. This is in qualitative agreement with our observations. The surface roughness is known to decrease the polarization, which is as observed with our data set; the regions where the wind is strong (or $\sigma_{0}$ is low) correspond to a small polarization. On the other hand, we can already eliminate the surface crust or layering effect, which would be intensified by the wind, because these phenomena increase the polarization (Stogryn, 1986); this is in contradiction to our observations.

In order to understand more quantitatively this correlation between wind intensity, $\sigma_{0}$ as measured by the altimeter and polarization of the microwave brightness temperature and emissivity, we will model the possible effects.

\section{(a) A model for TBv-TBh}

For TBv-TBh, we use the model of Stogryn (1986), deduced from the strong fluctuation theory. This model shows good agreement with the experimental data, without introducing correction factors as is necessary when using radiative transfer theory.

The electric field (or bistatic coefficient) is represented by the sum of a mean field (created by an effective medium having mean dielectric properties that depend only on depth) and of a random field of zero mean. The mean field is the sum of an incoherent field and of a reflected plane wave in air above the medium, proportional to the reflection coefficient of the effective mean medium ( $\mathrm{Ra}$, where $a$ is the polarization $h$ or $v$ ). The random field is characterized by a scattering coefficient $\gamma$, which depends on the directions of incident and scattered signals, and on their respective polarizations.

Then, the emissivity ea, in a given direction, can be written:

$$
\text { ea }=1-|\mathrm{Ra}|^{2}-1 /(4 \pi) \int\left(\gamma_{\mathrm{av}}+\gamma_{\mathrm{ah}}\right) \mathrm{d} \Omega
$$

where the integral is over the half-space above the surface. 
The last term increases with snow thickness. However, as shown by Stogryn (1986), at $37 \mathrm{GHz}$, it is almost constant when snow thickness reaches a few tens of meters. In the case of Antarctica, we will now consider this last term as dependent on volume scattering only. Therefore, we will write:

$$
\mathrm{ev}-\mathrm{eh}=\mathrm{Rh}^{2}-\mathrm{Rv}^{2}+\delta V S
$$

where $\delta V S$ is the difference in volume scattering for both polarizations:

$$
\delta V S=1 /(4 \pi) \int\left(\gamma_{\mathrm{vv}}-\gamma_{\mathrm{hh}}\right) \mathrm{d} \Omega .
$$

Hence, the polarization can be written as the sum of a "surface" part and a "volume" part.

\section{(b) Polarization due to volume effects}

In theory, as well as in practice, $\delta V S$ shows little dependence on grain-size or on temperature (Matzler and others, 1984; Stogryn, 1986). Also, volume scattering due to ice layers or to the surface crust are probably not very important; as a matter of fact, layers result either from melting and refreezing processes, or from wind action. First, the low temperature above $1000 \mathrm{~m}$ height prevents snow from melting. Secondly, as already mentioned, action of the wind would increase layering; the contrary being observed, one would assume that this mechanism can mostly be neglected compared to others, except perhaps for low $\sigma_{0}$ values (or strong winds) where we observed a different trend. If layers play a role in this case, they may create polarization effects, which can explain the behaviour of the polarization for low $\sigma_{0}$ values.

\section{(c) Polarization due to surface effects}

For a flat surface, the reflectivities can be obtained from the Fresnel law; for a jump of the dielectric constant of 1 in air to $\varepsilon$ in dry snow (the complex part of the dielectric constant is neglected), we can write:

$$
\begin{aligned}
& \mathrm{Rh}=\left(\left(\varepsilon-\sin ^{2} \theta\right)^{1 / 2}-\cos \theta\right) /\left(\left(\varepsilon-\sin ^{2} \theta\right)^{1 / 2}+\cos \theta\right) \\
& \mathrm{Rv}=\left(\left(\varepsilon-\sin ^{2} \theta\right)^{1 / 2}-\varepsilon \cos \theta\right) /\left(\left(\varepsilon-\sin ^{2} \theta\right)^{1 / 2}+\varepsilon \cos \theta\right)
\end{aligned}
$$

where $\theta$ is the observation angle, $50^{\circ}$ for the SMMR.

From Tiuri and others (1984), $\varepsilon$ depends only on the snow density $d$.

$$
\varepsilon=1+1.7 d+0.7 d^{2}
$$

A mean density of $0.4 \mathrm{Mg} \mathrm{m}^{-3}$ (Paterson, 1981) and a deduced dielectric constant of 1.8 (Equation (9)) lead to

$$
\begin{aligned}
& R v^{2}=0.0006 \\
& R h^{2}=0.069
\end{aligned}
$$

The vertical reflectivity $\mathrm{Rv}^{2}$ is therefore negligible. Remember that we indeed observed that the variations in polarization were mostly due to variations of the horizontal polarization.
We therefore find a theoretical value for ev-eh of the order of 0.07 , which is in agreement with measurements for dry snow in the Alps (Matzler, 1987), and with our observations (Fig. 3e).

A variation of the snow density from 0.35 to $0.45 \mathrm{Mg} \mathrm{m}^{-3}$ would imply variations of $\mathrm{Rv}^{2}$ from 0.06 to 0.08 . This is much smaller than the observed variations of eh. In addition, wind smashes the snow grains so that the snow density increases with wind intensity (Male, 1980); this would induce a correlation opposite to the observed one.

Similarly, wind would cool down the air temperature and lower the brightness temperature. Equation (4), which does not take into account the wind-cooling effect, will underestimate the emissivity. A $5 \mathrm{~K}$ cooling will induce a $2 \%$ error; this is quite negligible for ev-eh and of the order of -0.02 for ev or eh. This is also contrary to the observed general trend, but may explain the behaviour of 1-ev and 1-eh for small $\sigma_{0}$ (Fig. 5).

\section{(d) Polarization due to surface roughness}

The decrease of reflectivity with wind could be due to a surface-roughness effect. For example, at $35 \mathrm{GHz}$, ev-eh decreases from 0.08 to 0.035 when the snow is roughened by steepening (Matzler and others, 1984). We now look for a theoretical estimation of this surface-roughness effect.

The wind-induced surface features above Antarctica are of two kinds: micro-roughness on centimeter scales (Fung and Eom, 1982) and snow dunes or sastrugi, the vertical height of which is between 0.1 and $1 \mathrm{~m}$. At the wavelength of the SMMR $(0.9 \mathrm{~cm})$, micro-roughness should be the dominant factor affecting emissivity.

The effect of roughness on the surface part of emissivity is well known (Choudhury and others, 1979; Tsang and Newton, 1982), but, to our knowledge, no model exists for the effect of roughness on the volume part.

We will write:

$$
\mathrm{Ra}^{\prime 2}=\mathrm{Ra}^{2} \times f\left(S^{2}\right)
$$

where $S^{2}$ is the variance of the surface slope, $\mathrm{Ra}^{\prime 2}$ the modified reflectivity for polarization $a$.

When surface undulations are comparable to the wavelength, both coherent and incoherent reflectivities of the surface are affected (Tsang and Newton, 1982). Note that, when looking at the vertical, the incoherent part is dominant, as is the case for the altimeter (Equation (2)). The coherent part $f^{\mathrm{c}}$, which should mostly result from centimeter-scale roughness, is given in Choudhury and others (1979):

$$
f^{\mathrm{C}}\left(S^{2}\right)=\exp \left(-4\left(2 \pi \sin \theta \sigma_{\mathrm{h}} / \lambda\right)^{2}\right)
$$

where $\lambda$ is the wavelength of the signal of interest, $\theta$ is the look angle ( $50^{\circ}$ for SMMR), and $\sigma_{h}{ }^{2}$ is the variance of surface height, usually linked with the variance of the surface slope $S^{2}$ and correlation length $l$ as:

$$
\sigma_{\mathrm{h}}=s^{2} l^{2} / 2
$$

For larger undulations, the coherent part vanishes; the incoherent part, given in Tsang and Kong (1980), can be 
simplified in our case by:

$$
f^{\mathrm{i}}\left(S^{2}\right)=1-S^{2} / \sin ^{2} \theta .
$$

$S^{2}$ can be deduced from altimeter data (with Equations (1)-(2)). If we assume that the volume part is negligible, Equation (2) gives a maximum value of 0.01 for $S^{2}$. Then, only the coherent part $f^{\mathrm{C}}\left(S^{2}\right)$ plays a role. $f^{\mathrm{C}}\left(S^{2}\right)$ is represented, superimposed on the binned values in Figure $5 \mathrm{a}$ and c. We assume that $\delta V S$ and the volume part of the horizontal polarization are well averaged and take mean values of 0.02 and 0.12 , respectively. A very good fit to the observations is obtained for a correlation length $l$ of $1.5 \mathrm{~cm}$.

The larger features created by the wind are transparent to the altimeter return power because they do not greatly affect the reflectivity at the vertical. They can be superimposed on micro-roughness and modify the incoherent part of the reflectivity, at the SMMR observation angle. An important limitation of this study is the lack of measurements of the statistical parameters describing the surface roughness. In particular, a proportionality factor could be introduced into the definition of $S^{2}$ in Equation (2), depending upon the statistical law chosen for the surface description (Fung and Eom, 1982). For instance, the choice of an exponential law for the height distribution, rather than a random law, would increase $S^{2}$ by a factor 3 .

Nevertheless, it seems that the general trend of ev-eh and 1 -eh is well explained by the coherent perturbation term on the reflectivity due to surface roughness (Fig. 5). This is clear for $\sigma_{0}$ larger than $2 \mathrm{~dB}$. This suggests that the potential altimeter volume scattering is always smaller than this value; when the surface is smooth, the surface scattering hides the volume scattering and the relation between $\sigma_{0}$ and roughness is clear; one can assume that surface scattering dominates the altimeter echo. This corresponds to an AGC greater than $24 \mathrm{~dB}$, which is the case for $90 \%$ of the data (Remy and others, 1990).

In this case, the potential error on the measured altimetric height is small. This suggests, for the survey of the Antarctic topography, that one should select smoothsurface regions (by in-situ or spatial measurements) in order to avoid potential volume-scattering pollution when different maps are compared.

For the $10 \%$ remaining data, when the surface is very rough, volume scattering may be dominant, but we cannot easily conclude this. The theory predicts a lower polarization effect than the observed one. This may also be due to a layer effect as already mentioned in section IVb; this effect seems to be detectable for low $\sigma_{0}$ values, when the wind is strong, and creates a polarization effect. We also mentioned that wind cooling of the air (section IVc) or when the incoherent part is neglected (Equation (14)) may also explain the different behaviours. We have insufficient evidence to be able to reach a conclusion in this case.

Finally, if we assume that the correlation between brightness temperature and altimetric return is imputable to a roughness effect, one has to be careful when estimating accumulation rate over Antarctica from radiometric data. Strong winds will decrease the reflectivity and thus scattering (and the grain-size) will appear lower than the actual value (Equation (5)); accumulation rate will appear larger than its correct value. This may, perhaps, explain the low accumulation-rate value deduced by Rotman and others (1982) from passive radiometer data in Terre Adélie, where katabatic winds are known to be very strong (Parish, 1982).

\section{CONCLUSION}

The comparison between the intensity of the Seasat radaraltimeter return power (AGC) and passive microwave brightness temperature at $37 \mathrm{GHz}$ from the Nimbus-7 SMMR data shows little correlation. However, AGC is strongly correlated with polarization of the SMMR signal (correlation of 0.55 for 1250 domains of $25 \mathrm{~km} \times 25 \mathrm{~km}$ each). This correlation is still greater with polarization of emissivity, deduced from brightness temperature by normalization to in-situ temperature (correlation of 0.6 ). Assuming, after Matzler and others (1984), that volume scattering does not affect polarization much, it is found that the variations of polarization are mostly due to variations of horizontal reflectivity; the latter would result from variations in surface roughness, related to wind intensity. Using relations between the altimeter return power and the variance of surface slope, as well as relations of emissivity and this same parameter, the correlation between AGC and polarization at $37 \mathrm{GHz}$ can be well explained. This suggests that, except in areas with a very rough surface, the altimeter signal is dominated by surface scattering. This is a favorable situation for monitoring continental ice topography.

\section{REFERENCES}

Choudhury, B.J., T.J. Schmugge, A. Chang and R. W. Newton. 1979. Effect of surface roughness on the microwave emission from soils. 7. Geophys. Res., 84(C9), 5699-5706.

Colbeck, S. C. 1980. Dynamics of snow and ice masses. New York, etc., Academic Press.

Drewry, D.J. 1983. The surface of the Antarctic ice sheet. In Drewry, D.J., ed. Antarctica: glaciological and geophysical folio. Cambridge, University of Cambridge. Scott Polar Research Institute, Sheet 2.

Fung, A. K. and H.J. Eom. 1982. Application of a combined rough surface and volume scattering theory to sea ice and snow backscatter. IEEE Trans. Geosci. Remote Sensing, GE-20(4), 528-536.

Lettau, H. 1969. Antarctic atmosphere as a test tube for meteorological theories. In Quam, L. O., ed. Research in the Antarctic. Washington, DC, American Association for the Advancement of Science, 443-475. (Publication 93.)

Mätzler, C. 1987. Applications of the interaction of microwaves with the natural snow cover. Remote Sensing Rev., 2, 259-392.

Mätzler, C., R.O. Ramseier and E. Svendsen. 1984. Polarization effects in sea-ice signatures. IEEE $\mathcal{F}$. Oceanic Eng., OE-9(5), 333-338.

Parish, T. R. 1982. Surface airflow over East Antarctica. Monthly Weather Rev., 110, 84-90.

Partington, K. C., J. K. Ridley, C. G. Rapley and H.J. 
Zwally. 1989. Observations of the surface properties of the ice sheets by satellite radar altimetry. 7. Glaciol., 35(120), 267-275.

Paterson, W. S. B. 1981. The physics of glaciers. Second edition. Oxford, etc., Pergamon Press.

Remy, F., P. Mazzega, S. Houry, C. Brossier and J. F. Minster. 1989. Mapping of the topography of continental ice by inversion of satellite-altimeter data. $\mathcal{J}$. Glaciol., 35(119), 98-107.

Remy, F., C. Brossier and J. F. Minster. 1990. Intensity of satellite radar-altimeter return power over continental ice: a potential measurement of katabatic wind intensity. F. Glaciol., 36(123), 133-142.

Ridley, J. K. and K. C. Partington. 1988. A model of satellite radar altimeter return from ice sheets. Int. J. Remote Sensing, 9(4), 601-624.

Rotman, S. R., A.D. Fisher and D.H. Staelin. 1982. Inversion for physical characteristics of snow using passive radiometric observations. F. Glaciol., 28(98), 179-185.

Stogryn, A. 1986. A study of the microwave brightness temperature of snow from the point of view of strong fluctuation theory. IEEE Trans. Geosci. Remote Sensing, GE-24(2), 220-231.

Tiuri, M.E., A.H. Sihvola, E. G. Nyfors and M.T. Hallikainen. 1984. The complex dielectric constant of snow at microwave frequencies. IEEE F. Oceanic Eng., OE-9, 377-382.

Tsang, L. and J. A. Kong. 1980. Asymptotic solution for the reflectivity of a very rough surface. 7. Appl. Phys., 46, 5127-5133.

Tsang, L. and R. W. Newton. 1982. Microwave emissions from soils with rough surfaces. F. Geophys. Res., 87(C11), 9017-9024.

Zwally, H.J. 1977. Microwave emissivity and accumulation rate of polar firn. f. Glaciol., 18(79), 195-215.

Zwally, H.J., R.A. Bindschadler, A.C. Brenner, T.V. Martin and R.H. Thomas. 1983. Surface elevation contours of Greenland and Antarctic ice sheets. F. Geophys. Res., 88(C3), 1589-1596.

The accuracy of references in the text and in this list is the responsibility of the authors, to whom queries should be addressed. 